J. Astrophys. Astr. (1994) 15, 357-385

Reproduced from Proceedings of the Indian Academy of Sciences Vol. A2, 190-200, 1943

\title{
FLUCTUATIONS OF LIGHT INTENSITY IN CORONE FORMED BY DIFFRACTION
}

\author{
By G.N. RAMACHANDRAN \\ (From the Department of Physics, Indian Institute of Science, Bangalore) \\ Received September 22, 1943 \\ (Communicated by Sir C.V. Raman, Kt., F.R.S., N.L.)
}

\section{Introduction}

THE problem of determining the resultant of the secondary radiations emitted by a cloud of similar particles under the action of a primary wave is an important one in the field of optics. It might be supposed that when there are a large number, $n$, of such particles, distributed at random, the resultant intensity is just $n$ times that due to a single particle. The late Lord Rayleigh (1871), however, showed that this is far from being the case, and that the resultant in any particular trial may be anywhere between $o$ and $n^{2}$, and does not show any tendency to close upon the value $n$. He has also investigated (1880) the probability that the resultant intensity may lie between assigned limits of magnitude, and has derived an expression for the distribution of the resultant intensity.

In a general way, the existence of such fluctuations of intensity is illustrated by the well-known experiment of using a glass plate on which lycopodium powder is dusted as the diffraction screen, and viewing a source of light through it. The effects observed depend greatly on the size of the source and its spectral nature. Even as early as 1877, Exner observed the existence of radial streaks such as those shown in Fig. 3(a), Plate I, when the corona is seen in white light. More recently, Laue $(1916,1917)$ and de Haas (1918) have observed the fluctuations in monochromatic light. In this case, the radial fibres reduce to a "mottled structure" of the 
type shown in Fig. $3(b) .^{\star}$ Laue's studies led him to suppose that the phenomena could not be completely explained on the basis of classical wave-optics. This contention was disputed by de Haas, who performed further experiments supporting the view that the phenomena are explicable on wave-principles.

In this paper, some theoretical considerations are presented regarding the nature of the fluctuations, and experiments performed with a view to substantiate the theory are also described. It is found, as is shown below, that de Haas's explanation of the phenomena is inadequate, and does not go to the root of the problem. In particular, the Rayleigh statistical law of distribution of intensity has been quantitatively verified for the first time, making use of the fluctuations of intensity in the corona as the basis for measurement.

2. The Nature of the Fluctuations in Monochromatic Light

de Haas's explanation of the mottled structure consists in considering every pair of particles to give rise to a set of interference fringes crossing the field. These are superposed, and produce fluctuations of intensity. This explanation, however, is wholly inadequate. In fact, when the number of particles is very large, one must take the aggregate effect of all the particles into account and not merely the effect due to pairs of particles. Each particle gives rise to a corona of its own, and the resultant intensity in any direction is due to the collective action of the wave-fronts diffracted by all the particles in that direction.

The failure of the de Haas point of view may be brought out strikingly by the following analogy. Suppose we are considering the opposite case of a regular square diffraction grating, formed for example by a square mesh of wires. In this case, one may take each pair of meshes in the grating, and imagine it as giving rise to a set of interference fringes of the type imagined by de Haas. But, such a method gives us no idea at all of what the nature

* This will hereafter be referred to also as the "fine-structure" or "structure” of the corona. 
of diffraction pattern due to the complete grating would be. The latter could be determined only by taking the waves diffracted by all the meshes simultaneously into account and finding their total effect. In those directions in which all these waves reinforce, we get bright spots, while elsewhere we have dark areas.

In the same way, with the lycopodium powder also, one has to consider the waves diffracted by all the particles together. Each particle becomes the centre of secondary radiation, and in those directions in which the phase relations between the waves diffracted by the particles happen to be such that there is a large co-operative effect, there will be bright spots. Since the positions of the particles on the screen are unknown, one cannot determine the positions of these maxima of intensity; but it is easily seen that there must be a large number of such spots irregularly arranged in the field of view.

We now proceed to show that these spots are in fact as sharply defined as the original image of the source formed by a lens whose aperture is the same as that of the diffracting screen. Consider a point $P$ in the focal plane of the camera where due to the cooperative action of the waves from the various particles, we have a large intensity, say $I_{P}$. The amplitude at this point will be $u_{\mathrm{P}}=\sum_{1}^{n} e^{i p_{m}}$ where $p_{m}$ is the phase of the $m$ th particle on the screen, and the summation is done for all the $n$ particles present, it being assumed that the amplitude due to each particle is unity. Therefore,

$$
\mathrm{I}_{\mathrm{P}}=\left(\sum_{1}^{n} \cos p_{m}\right)^{2}+\left(\sum_{1}^{n} \sin p_{m}\right)^{2}=n+\sum_{r=1}^{n} \sum_{s=1}^{n} \cos \left(p_{r}-p_{s}\right) .
$$

Consider now a neighbouring point $\mathrm{P}^{\prime}$. At this point, the phases of the waves from the various particles would be altered. Let the alteration of phase for the $m$ th particle be $\delta_{m}$. Then, the 
amplitude at $\mathrm{P}^{\prime}$ is

$$
\begin{aligned}
& u_{P^{\prime}}=\sum_{1}^{n} e^{i p_{m}} e^{i \delta_{m}}, \text { and the intensity is } \\
& \mathrm{I}_{\mathrm{P}^{\prime}}=\left[\sum_{1}^{n} \cos \left(p_{m}+\delta_{m}\right)\right]^{2}+\left[\sum_{1}^{n} \sin \left(p_{m}+\delta_{m}\right)\right]^{2} .
\end{aligned}
$$

This can be expressed in the form

$\mathrm{I}_{\mathrm{P}^{\prime}}=n+\sum_{r} \sum_{s} \cos \left(\delta_{r}-\delta_{s}\right) \cos \left(p_{r}-p_{s}\right)+\sum_{r} \sum_{s} \delta_{s} \sin \left(p_{r}-p_{s}\right)$.

If the $\delta$ 's are small, this can be put in the form

$$
\begin{aligned}
\mathrm{I}_{\mathrm{P}^{\prime}}=\left[n+\sum_{r} \sum_{s} \cos \left(p_{r}-p_{s}\right)\right] & +\sum_{r} \sum_{s} \delta_{r} \delta_{s} \cos \left(p_{r}-p_{s}\right) \\
& +\sum_{r} \sum_{s} \delta_{s} \sin \left(p_{r}-p_{s}\right) .(3)
\end{aligned}
$$

The second term is negligible, since it involves second order terms, so that

$$
\mathrm{I}_{\mathrm{P}^{\prime}}-\mathrm{I}_{\mathrm{P}}=\sum_{r=1}^{n} \sum_{s=1}^{n} \delta_{s} \sin \left(p_{r}-p_{s}\right) .
$$

Now, $\sin \left(p_{r}-p_{s}\right)$ is of the same order of magnitude as $\cos \left(p_{r}-p_{s}\right)$, so that $\sum \sum \delta_{s} \sin \left(p_{r}-p_{s}\right)$ is of a lower order compared with $\sum \sum \cos \left(p_{r}-p_{s}\right)$ which is the order of $\mathrm{I}_{\mathrm{p}}$. Thus, if $\delta$ is small, $\left(\mathrm{I}_{\mathrm{P}^{\prime}}-\mathrm{I}_{\mathrm{P}}\right)$ is small compared with $\mathrm{I}_{\mathrm{P}}$.

Now, if we denote by $\delta \phi$ the angular separation of $\mathbf{P}$ and $\mathbf{P}^{\prime}$, then the largest value of $\delta_{r}$ or $\delta_{s}$ is given by $\pi b \delta \phi / \lambda$, where $b$ is the width of the diffraction screen, and $\lambda$ the wave-length of light. It is evident from (4) that $I_{P^{\prime}}$ does not sensibly differ from $I_{P}$ over a range of values of $\delta \phi$ within which the largest of the $\delta$ 's does not exceed a fraction of $2 \pi$, and over this range of angles, the large intensity at $P$ will persist. Putting the maximum value of the $\delta$ 's for this to happen as, say, $\pi / 4$ one gets the width of the bright spot as

$$
\delta \phi=\frac{1}{4} \frac{\lambda}{b}
$$


Thus, the angular width of the bright spot is of the order of $\lambda / b$. Now, it is well known that the diffraction image due to a lens of aperture $b$ also extends over an angle of the order of $\lambda / b$, so that the bright spot at $\mathrm{P}$ will be of the same sharpness as an image of the source formed by a lens of aperture $b$.

The argument used in the above discussion is, however, not restricted to the case when the intensity at $P$ is a maximum. Actually, it is true whatever may be the intensity at $P$, so that one sees that the corona must actually consist of spots whose extension is of the same size as that of the image of the source. We have proved above that these images would not be spread out by an extent more than the aperture of the lens, so that we must expect the fine-structure to consist of sharp and well-defined images of the original source distributed at random. They are in fact the spectra formed by the irregular arrangement of the sources of secondary radiation produced by the particles.

Another interesting result, regarding the number of such spots in the field of view, comes out of the above discussion. As already remarked, the extent of the individual spots is given by the width of the aperture. Thus, we may divide the whole field of view into a number of areas, each of which is occupied by one spot. Now, on increasing the aperture, the area occupied by a spot decreases, so that more spots must appear in the field of view. To observe this phenomenon, the source of light must be small enough, so that the spots do not sensibly overlap. Otherwise, the fluctuations of intensity would all be wiped out by the overlapping, and the individual spots could not be discriminated. Under favourable conditions, however, the argument shows that the density or the number of spots per unit area must depend on the aperture employed, increasing with increase of its dimensions.

\section{Experimental Confirmation}

The verification of the above two deductions from the theory was done by the following experimental arrangement. The source of light, S (Fig. 1) was a pinhole, or an aperture of any size and 
shape as desired, which was illuminated by the filtered mercury radiation of $5461 \mathrm{~A}$. U. At a certain distance from the source was placed a glass plate $\mathrm{G}$, on which lycopodium powder was dusted. The resulting diffraction pattern could be brought to a focus on a plate $C$ by a lens $L$. An aperture $A B$ was placed in front of the lens, and its size and position could be adjusted. When necessary, the camera could be removed and the pattern directly viewed with the eye.

Initial visual observations using a small circular hole as the source showed that the spots in the corona are also all circular in shape and of the same size as the original source. On increasing or decreasing the size of the pinhole, the spots in the field of view also correspondingly became larger or smaller showing that they are images of the source. But the most convincing proof of this fact was obtained by using a triangular slit as the source, when every one of the spots assumed a triangular shape, and besides had the same size and orientation as the source. Photographs were then taken to illustrate this fact. They are reproduced in Fig. $4(a)$ and (b), Plate I. Fig. $4(a)$ was taken with a circular pinhole as the source, and Fig. $4(b)$ with a triangular slit. They show in an unmistakable manner that the spots are in reality images of the source.

The second conclusion from the theory was also verified by observation. On increasing the area of the aperture, it was found that the number of spots in the field of view increased, and vice versa as demanded by the theory.

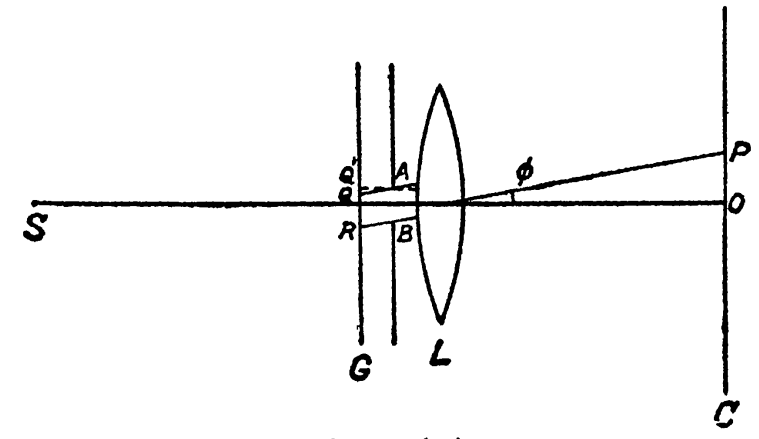

Fig. 1. Experimental Arrangement 
During these experiments, a few other interesting phenomena were observed which also find a ready explanation on the idea that the spots are the spectra produced by the collective diffraction by all the particles.

(a) If one moves the screen containing the lycopodium powder keeping the eye fixed on the source, then the ring system is not found to undergo any change, but the fine-structure appears to move relative to the pattern of rings in the same direction as the motion of the screen. Vice versa, keeping the screen fixed, if one moves the eye, all the while looking at the source, then the finestructure appears to move in a direction opposite to the motion of the eye. This effect is only the converse of the previous one. Also, if the screen is rotated, the fine-structure appears to rotate in the same direction.

These effects can be explained as being purely due to the geometry of the arrangement. Since the pupil of the eye is small, the whole of the screen does not contribute to the diffraction pattern nor is every portion of the pattern produced by the same portion of the screen. If we consider the corona in a direction $\phi$ (Fig. 1), the intensity in this direction is due only to the waves diffracted by the region QR of the screen. Hence, if the screen is moved up, the same portion $\mathrm{QR}$ is now responsible for the corona in a direction $\phi^{\prime}$ parallel to $\mathrm{Q}^{\prime} \mathrm{A}$. This happens for every portion of the screen, so that the fine-structure as a whole appears to move in the same direction as the screen relative to the rings. The effect of rotation is too obvious to need an explanation.

(b) During these experiments, it was found that the fine-structure not only moved bodily in the same direction as the motion of the screen, but that there were some internal changes as well. To follow these, the bodily motion was avoided in the following way. The aperture AB was placed in contact with the screen, and the lens $L$ of the camera was large enough so that the whole diffraction pattern was caught by it. In this way, the difficulty of different portions of the screen giving rise to different portions of the corona was overcome. The whole of the screen was always 
operative. Now, on moving the screen in its own plane, the finestructure pattern at the focal plane of the lens did not show any bodily movement; but there was an internal rearrangement of the spots.

This phenomenon could be explained as due to the fact that spherical waves are incident on the screen so that, on moving the screen in its own plane, the phase of the wave falling on each particle is altered. Thus, the phase relations between the waves emitted by different particles are changed, which leads to a change in the pattern of the spectra given by the particles and hence to a random rearrangement of the spots.

It is obvious that these internal changes must vanish if the incident wave is plane, for then a movement of the plate in its own plane should produce no change in the phase relationship between the different particles. This was tested out experimentally and it was found that with parallel light incident on the screen, the fine-structure pattern at the focal plane of the lens was entirely unaffected by moving the screen in its own plane. This shows that the explanation given above for the internal motion of the spots is correct.

(c) The phenomenon of the internal rearrangement of the spots can be observed with the eye also, if the aperture $A B$ is made sufficiently small. In this case, on moving the aperture over the screen, different portions of the screen are operative, and since the arrangement of the particles in these are different, the spots in the field of view exhibit a beautiful "dance". The appearance is somewhat similar to that of the field of a spinthariscope, with bright spots appearing and disappearing at random positions.

\section{Fluctuations in White Light}

We have already shown how, in monochromatic light, the finestructure consists of a series of images of the source. We now proceed to investigate what happens if white light is used. Suppose that, with monochromatic light of wavelength $\lambda$, there is a bright spot at $\mathrm{P}$, in the direction $\phi$ (Fig. 1). In the diffracting screen, 
draw the $\mathrm{X}$-axis parallel to $\mathrm{OP}$, and the $\mathrm{Y}$-axis perpendicular to it, and let $\left(x_{m}, y_{m}\right)$ be the co-ordinates of the $m$ th particle on the screen with respect to these axes of co-ordinates. Then, (supposing the incident wave to be plane) the intensity in the direction $\phi$ is equal to

$$
\left|\sum_{m=1}^{n} e^{i k x_{m}} \sin \phi\right|^{2}
$$

where $k$ stands for $2 \pi / \lambda$. Now, supposing the wavelength of the light to be changed to $\lambda^{\prime}$, and that $k^{\prime}=2 \pi / \lambda^{\prime}$, then it is readily seen from the above expression that the value of the intensity is unaltered provided we change $\phi$ to $\phi^{\prime}$, such that

$$
k \sin \phi=k^{\prime} \sin \phi^{\prime}, \text { or } \sin \phi / \lambda=\sin \phi^{\prime} / \lambda^{\prime} .
$$

Hence, the bright spot, which was formerly at $\mathrm{P}$, would now appear at another point $\mathrm{P}^{\prime}$ in the direction $\phi^{\prime}$ given by expression (7) such that $\mathrm{P}^{\prime}$ lies along OP. This argument also is not restricted to the case when $\mathrm{P}$ is a bright spot, but is quite general. Hence, with an increase in the wavelength of the light, the whole pattern expands radially so that to every point $\mathrm{P}$ in the first case there is one, $\mathrm{P}^{\prime}$, in the second, such that

$$
\frac{\mathrm{OP}}{\lambda}=\frac{\mathrm{OP}^{\prime}}{\lambda^{\prime}} .
$$

If white light is used, there will be a range of wavelengths, so that there will be no discrete spots, but each spot will be spread out into a radial streak, whose colour is violet at the inner end and red at the outer end. The length of every such streak will be directly proportional to its distance from the centre, the streaks further away from the centre being longer.

As already remarked, such radial fibres were actually observed in white light by Exner. The author has observed these carefully and has found that one can actually follow the spectral colours in the order violet to red in any single fibre, as one goes away from the centre. Very close to the centre of the field, however, one can 
observe a few white spots, since the dispersion in them is very small on account of their proximity to the centre. The length of the fibres (the distance from the violet to the red end) is found to be proportional to their distance from the centre, and the length of any fibre at a certain distance is found to be a constant, within the limits of the experimental error.

In order to further demonstrate the correlation between the spots in monochromatic light, and the fibres in white light, the following experiment was performed. A pointolite lamp was used to illuminate a fine pinhole, and a mercury green filter supplied by Adam Hilger \& Co., was inter ${ }_{r}$ osed. This transmitted only two bands, one in the green and the other in the red. The finestructure now presented a beautiful appearance. The pattern was filled with red and green spots; but every green spot was accom: panied by a red one, the two being along a radius, and the red spot being the outer one. It was also verified that the ratio of the distances of the red and the green spots from the centre was a constant, and was equal to the ratio of the mean wavelengths of the red and green transmission bands.

\section{Verification of the Rayleigh Law of Fluctuations}

Lord Rayleigh (1880) derived an expression for the distribution of intensity in the resultant due to $n$ vibrations whose phases are at random. If the amplitude of a vibration is taken as unity, and as a special case, if the phases are assumed to have only either of values 0 or $\pi$, then it follows directly from Bernoulli's theorem that the chance of a positive amplitude between $x$ and $x+\delta x$ is $\frac{1}{\sqrt{2 \pi n}} e^{-x^{2} / 2 n} \delta x$. In the more general case of arbitray phase, the probability that the resultant amplitude may lie between $r$ and $r+\delta r$ comes out as

$$
\frac{2}{n} e^{-r^{2} / n} r \delta r
$$

From this, it follows that the probability that the intensity may be between $\mathrm{I}$ and $\mathrm{I}+\delta \mathrm{I}$ is

$$
p(\mathrm{I}) \delta \mathrm{I}=\frac{1}{n} e^{-\mathrm{I} / n} \delta \mathrm{I}
$$


We can put this in a more convenient form. From (10), it can be shown that the average value of the intensity is $n$. Expressing I as a fraction $f$ of the average value, i.e., putting $\mathrm{I} / n=f,(10)$ reduces to

$$
p(f) \delta f=e^{-f} \delta f .
$$

This gives the probability that the resultant intensity may lie between the fractions $f$ and $f+\delta f$ of the average, and is the same function for all values of $n$. It is a maximum for $f=0$, and decreases as $f$ increases.

This law can be verified by making use of the fine-structure in the corona. As already said, the fine-structure is produced as a result of the interference of the light diffracted by all the powder grains. The relative phases of these diffracted waves are not known but are distributed at random. Also, for different angles, $\phi$, the phase relations are different, so that the whole pattern actually presents the effect of different combinations of these phase relations. Hence, the resultant intensity in the pattern must show the same statistical law of fluctuations as demanded by Rayleigh's theory.

This was verified in the following way. A very fine pinhole illuminated by the mercury green radiation of $5461 \AA$.U. was used as a source of light, and a picture of the central portion of the corona was taken with an exposure of two hours on a Selochrome plate. To obtain a standard of intensities, a square grating was used instead of the lycopodium screen, and the same exposure was given for the grating also. The grating gave a spectrum, the intensities of whose spots are calculable. The plates for the two photographs were obtained by cutting a single quarter plate into two. Both were developed simultaneously in the same developing bath for the same time. Thus, a strictly comparable scale of intensities was obtained for determining the intensity of the spots in the corona. The photographs of the corona and the standard grating spectra are reproduced in Fig. $5(a)$ and $5(b)$, Plate I, respectively. 
Now, in the corona, the average intensity falls away from the centre in the proportion $\mathrm{J}_{1}{ }^{2}(x) / x^{2}$ where $x=2 \pi a \sin (\phi) / \lambda$, $a$ being the radius of the particle, $\phi$ the angle of the diffraction, and $\lambda$ the wavelength of the light. The corona taken in the present case extended upto a value of $x=1.5$, for which the average intensity was about half that at the centre. It was therefore divided into annular rings, the widths of which were all equal to $x=0.25$, by drawing circles of radii corresponding to $x=0.25,0.5$ etc.. Within each ring, the value of $\mathrm{J}_{1}{ }^{2}(x) / x^{2}$ did not vary much, and the average value for a ring was taken to be correct for that ring.

Using the grating spectrum, the intensity of whose spots could be calculated, an arbitrary scale of intensities was obtained. Then, the spots in each ring were classified into this scale, comparing them visually with the standard. The spots having an intensity less than 1 in the arbitrary scale, i.e., those that could not be differentiated with the eye were found by calculating the total number of spots (dividing the area of the ring by the area of a spot) and subtracting from this the number of counted spots. Using this tabulated list, the average intensity (on the arbitrary scale) for that ring could be determined. Thus, the fraction of the total number of spots having an intensity between the fraction $f_{1}$ and $f_{2}$ of the average could be found. Dividing this by $\left(f_{1}-f_{2}\right)$ we get the value of $p(f)$ at $\left(f_{1}+f_{2}\right) / 2$. Thus, $p(f)$ was evaluated for certain values of $f$. This was done for four rings, viz., $x=0.25$ to 0.50 , to 0.50 to $0.75,0.75$ to 1.00 , and 1.00 to 1.25 . These were plotted in a graph and are reproduced in Fig. 2. The continuous curve represents the theoretical curve calculated from theory, and the experimental values are plotted by points.

A final average of all these four rings was obtained by dividing the intensities of the spots in each by the value of $\mathrm{J}_{1}{ }^{2}(x) / x^{2}$ for it, and then determining $p(f)$ and $f$ taking them all into account. These are tabulated in the table below, and are represented by black dots in Fig. 2. It is clearly seen that the experimental points fit the theoretical curve very well, considering 
Fluctuations of Light Intensity in Coronce

\begin{tabular}{c|l|c|c}
\hline$f$ & $p(f)$ & Theoretical & Difference \\
\hline 0.44 & 0.60 & 0.62 & -0.02 \\
1.29 & 0.33 & 0.28 & +0.05 \\
2.13 & 0.14 & 0.12 & +0.02 \\
3.45 & 0.02 & 0.03 & -0.01 \\
6.25 & 0.002 & 0.002 &.. \\
\hline
\end{tabular}

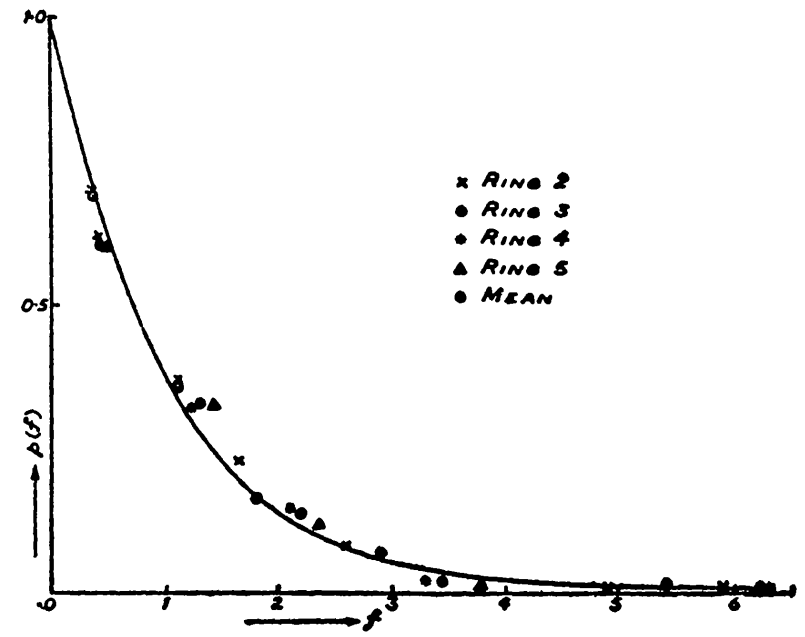

Fic. 2. Verification of Rayleigh's Statistical Law

that the comparison of intensities was done visually. Rayleigh's formula is therefore verified to be true for the distribution of intensity in the corona.

\section{Conclusion}

The above considerations regarding the fluctuations of intensity are true only for a static diffraction screen, i.e., one in which the phase relations between the various particles is fixed in time. If the particles themselves are in motion, as in a gas or a cloud, then a continuous redistribution of phase takes place, and what one sees is the integrated effect of all these over a definite period. As Lord Rayleigh (1918) has shown, such a redistribution tends 
to make the resultant intensity (perceived as an average over a certain period) approach the average value $n$, this tendency being greater the larger the number of redistributions. Hence, for the light scattered by a gas, or a cloud of particles, one is quite justified in regarding the scattered intensity in inclined directions as equal to $n$ times that due to a single particle.

If the motion of the particles is slow, then the alteration in the position of the spots in the fine-structure will also be slow, and can be observed. Such a slow random motion takes place in Brownian movement, so that it must be capable of detection by this technique. This possibility, which was suggested by Prof. Sir C.V. Raman, is now under investigation.

I wish to express my sincere thanks to Prof. C.V. Raman for suggesting the problem, and for the many helpful hints he gave during the investigation.

\section{Summary}

The fluctuations of light intensity in the diffraction corona produced by a large number of randomly distributed particles are investigated both theoretically and experimentally. The theoretical considerations show that the view put forward by de Haas is inadequate and that really the fluctuations arise owing to the interference of the waves diffracted by all the particles, which gives rise to a large number of sharp images of the source in monochromatic light. In white light, these spots must spread out, and produce radial streaks. These deductions from theory are all borne out by experiment.

Making use of the fluctuations of intensity in monochromatic light, verification has been made for the first time of Rayleigh's statistical law of distribution of intensity in the resultant of $n$ vibrations of arbitrary phase. It is shown that the resultant intensity shows no tendency to close upon the average value, $n$, but that it is distributed over a wide range of values. 


\section{REFERENCES}

1. de Haas, W.J. .. Proc. K. Akad., Amsterdam, 1918, 20, 1278.

2. Exner

.. Sitzungsher, Akad. Wien, 1877, 76, 522.

3. Laue, von

.. Phys. Gesselsch., Züurıch, 1916, 90.

4. _.. Berichte deutsch. Phys. Gesselsch, 1917, 19, 19.

5. Rayleigh, Lord .. Proc. Lond. Math. Soc., 1871, 3, 267.

6. .. Phil. Mag., 1880, 10, 73.

7. .. Ibid., 1918, 36, 429. 

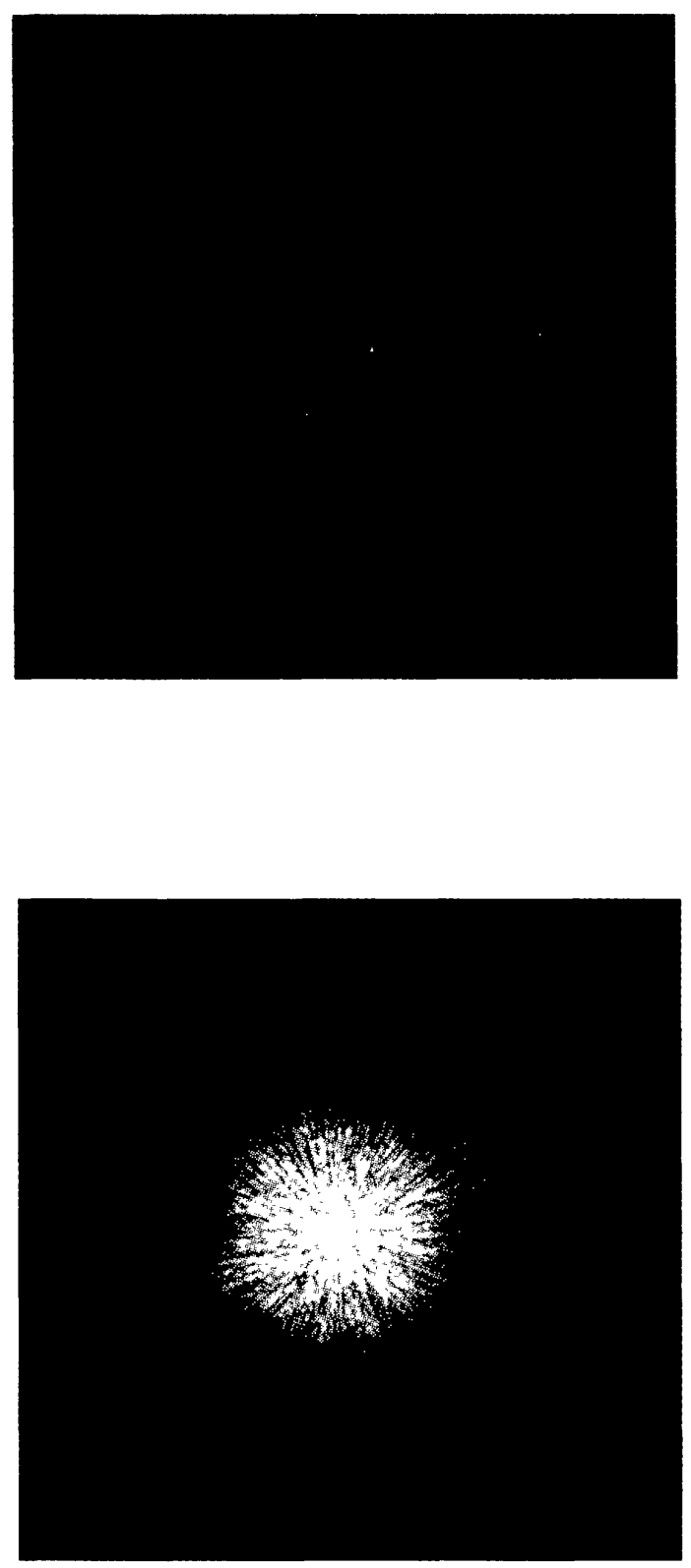

Speckles in monochromatic (top) and white light (bottom). [Photographs courtesy of Prof. R.P. Riesz]. We refer to a description of the speckle pattern in white light in page 9 and 10 of G.N. Ramachandran's paper. 
G. N. Ramaihandran Proc. Ind. Acad. Sci., A, zol. A $1 / 11$, H. I

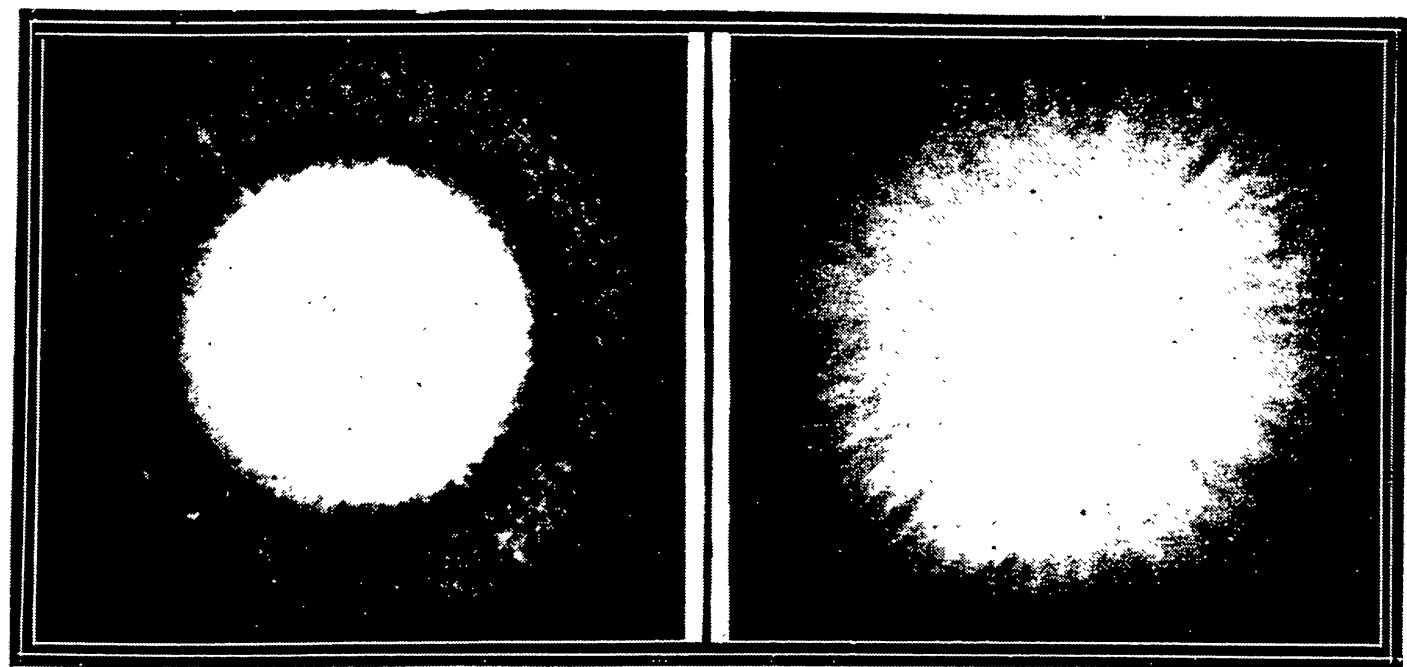

FIG. 3(a)

firc. $3(b)$

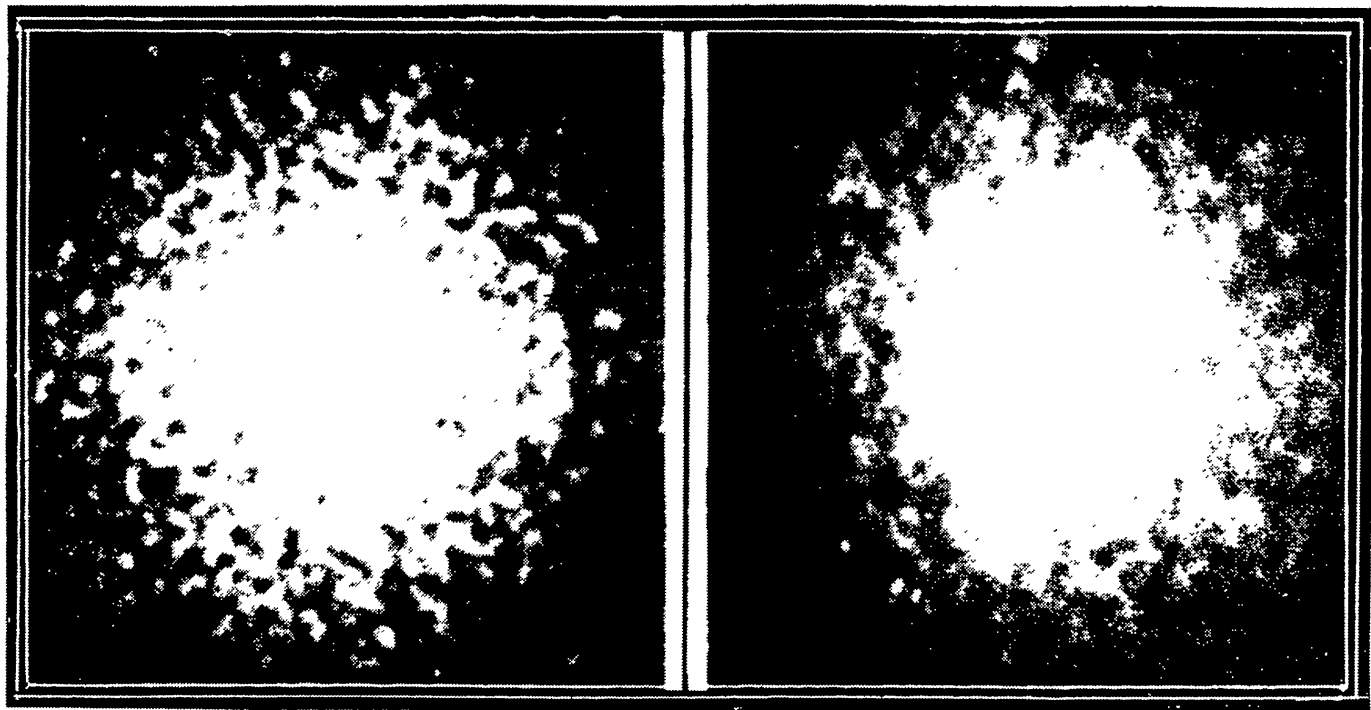

Frg. $4(a)$

Fick. $4(6)$

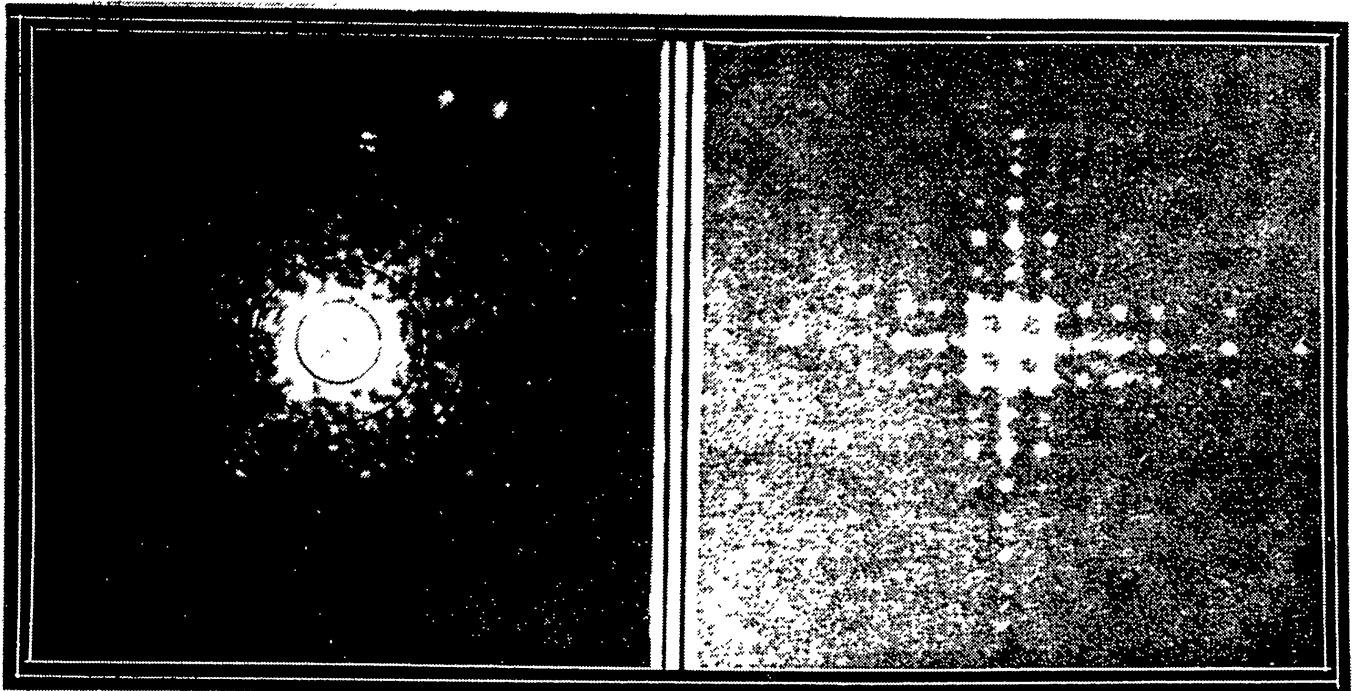

FIG. $5(a)$

Fif 5 . $5(b)$

(C) Indian Academy of Sciences - Provided by the NASA Astrophysics Data System 